\title{
Perspectives of a catheter-based nitric oxide sensor for the evaluation of endothelial function
}

\author{
Shichiro Abe and Teruo Inoue
}

\section{Key words:}

Endothelial function, Nitric oxide, Flow-mediated dilation, Nitric oxide sensor

\section{Introduction}

Vascular endothelial cells, which are considered to be the largest endocrine organ, secrete various vasoactive agents including nitric oxide (NO), to maintain homeostasis of the blood vessel wall. In particular, NO not only diffuses into underlying smooth muscles, causing them to relax and regulate vessel diameter, but also regulates inflammatory cell adhesion, vascular permeability, and coagulation via the fibrinogenolysis system. Thus, NO is regarded as an antiatherosclerotic agent, and such homeostatic roles represent the fundamental "endothelial function". Endothelial dysfunction is the initial step for vascular failure leading to cardiovascular events ${ }^{1)}$. Namely, the quantity of NO produced by vascular endothelial cells via the endothelial nitric oxide synthase (eNOS) directly reflects endothelial function. However, the quantitative measurement of NO is very difficult, because the life span of NO is very short. Alternatively, flow-mediated dilation (FMD) has been highlighted as a method to assess NO-dependent endothelial function. The FMD is widely used as an increasing of the diameter of the target artery, which is measured by high-resolution external vascular ultrasound in response to the increase in blood flow that occurs during ischemic reactive hyperemia. Reperfusion during FMD measurement increases shear stress on endothelium. Therefore, NO released from endothelial cells in response to increased shear stress diffuses into smooth muscles to promote relaxation of vasomotor tone, and consequently, the artery becomes dilated ${ }^{2,3)}$. However, NO bioavailability is not directly represented by the process of ischemia to reactive hyperemia in FMD measurement, because the FMD depends on, not only the release of endothe- lial NO-release, but also other conditions of the target artery, including smooth muscle function.

Circulating NO is assessed by the measurement of plasma levels of $\mathrm{NO}_{x}$, i.e., a composite of nitrite $\left(\mathrm{NO}_{2}^{-}\right)$and nitrate $\left(\mathrm{NO}_{3}^{-}\right)$, which are stable metabolites of $\mathrm{NO}^{4}$. However, the $\mathrm{NO}_{x}$ reflects not only eNOS-derived NO but also NO synthesized by the neuronal or inducible NOS isozymes, so does not directly represent endothelial function alone. On the other hand, plasma levels of asymmetric dimethylarginine (ADMA), known as an eNOS inhibitor, are also often measured. In general, ADMA competitively inhibits the activity of eNOS and reduces the production of NO in endothelial cells; thus, increased ADMA levels are thought to represent endothelial dysfunction ${ }^{5}$. However, measurement of these biomarkers cannot provide information about the kinetics of circulating endothelial cell-derived NO in real-time.

\section{Catheter-based NO measurement system}

Recently an in-vivo direct measurement system for circulating blood NO concentrations using a catheter-based NO sensor has been developed ${ }^{6}$. With this system, several investigators have attempted to evaluate in-vivo NO bioavailability in real-time ${ }^{7-12)}$ in the blood stream. This system is comprised of an amperometric NO-selective sensor (amino-700 XL, Innovative Instruments, Tampa, FL, USA), bonded at the tip of a 4-Fr catheter with a monorail lumen $(1200 \mathrm{~mm}$ long; Hirakawa Hewtech, Ibaraki, Japan). Polyurethane was attached to the detection tip to prevent physical damage of the vessel wall, and two metal wires were attached along the detection tip to provide mechanical support for the elec-

Department of Cardiovascular Medicine, Dokkyo Medical University

Corresponding author: Shichiro Abe, abenana@dokkyomed.ac.jp

Received: November 9, 2018, Accepted: December 17, 2018

Copyright (C) 2019 Japan Society for Vascular Failure 

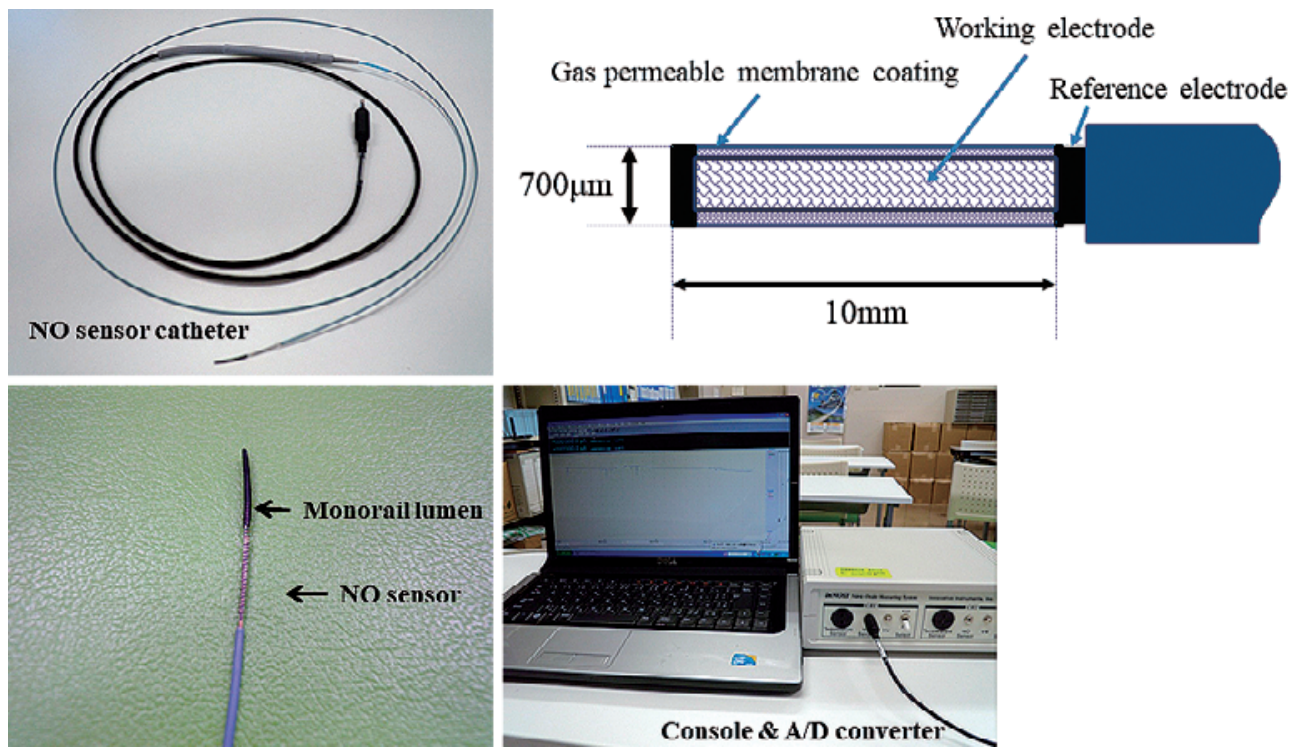

Figure 1. NO sensor catheter system.

trodes. The oxidative current produced by NO is monitored using a NO monitoring system (model in NO-T, Innovative Instruments) (Figure 1). Both the working and reference electrodes are integrated in a single element. The sensing electrode is covered with a $\mathrm{NO}$ gas permeable membrane. Thus, the NO gas diffuses through the electrode surface causing an electrical current that is monitored and recorded after A/D conversion in the interface. The sensor does not measure the absolute levels of circulating NO, because it measures the electrical potential differences between the reference site and the monitoring site, and therefore, converts milliampere to nanomole (nM) according to a previouslyinstalled calibration scale.

\section{In-vivo circulating NO kinetics during ischemia/reactive hyperemia}

At present, the in-vivo NO kinetics during the FMD procedure have not been proven. We hypothesized that a novel maneuver for the direct-estimation of NO kinetics using the catheter-based NO measurement system in the endothelial response to ischemia/reactive hyperemia could be used to evaluate the conformity of FMD. Therefore, we measured circulating NO levels in the forearm artery directly using this system.

To measure circulating NO levels, we deployed the NO sensor catheter into the forearm arteries of two patients with coronary artery disease, during diagnostic coronary angiography procedures, which were performed on the day after the FMD measurement, in 2010. These procedures were performed after approval of the hospital ethical review committee, and written informed consent was obtained from each of the two patients. Following insertion of a sheath by the radial approach, the NO sensor catheter mounted on a 0.014inch guidewire with the occlusion balloon (PercuSurge
GuardWire $^{\circledR}$; Medtronic Inc.) was positioned in the brachial artery. In this study, we generated an ischemia/reactive hyperemia model, similar to the FMD maneuver, by inflation/ deflation of the occlusion balloon at the proximal site of the catheter-tip NO sensor. At the distal site, a pressure/flow wire (Combo-wire ${ }^{\circledR}$; VOLCANO U.S.) was positioned for continuous monitoring of blood pressure and blood flow. The time required to obtain a high-quality baseline scan was over $5 \mathrm{~min}$. The balloon was inflated for $5 \mathrm{~min}$ until the blood pressure and blood flow disappeared in downstream monitoring, and following a deflation period of $2 \mathrm{~min}$, in succession. This ischemia/reactive hyperemia model was based on the procedure for FMD measurement. Throughout this process, circulating NO levels were monitored every 5 sec continuously using the NO sensor catheter (Figure 2). The FMD measurement was performed, according to a previously reported method ${ }^{3)}$. The normal FMD value was regarded as $>6.0 \%{ }^{13)}$.

The first case was a 69 year-old male with a normal FMD value of $6.9 \%$. In this case, the baseline NO level was 52.2 $\mathrm{nM}$. After occlusion of the vessel by inflation of the balloon, the NO level increased immediately and remarkably to the peak value of $76.4 \mathrm{nM}$ (46\% increase) and then gradually recovered to the baseline level. Following balloon deflation, the NO level increased gradually and slightly to 55.2 $\mathrm{nM}(5.7 \%$ increase) and then returned to the baseline level (Figure 3-A). We speculate that the immediate and transient NO elevation after balloon inflation might be caused by hypoxic stress or change of shear stress accompanied with occlusion of the blood flow. We also speculate that the gradual secondary elevation of NO levels, might be a part of the response to reactive hyperemia. We consider that these circulating NO kinetics might be representative in cases in which vascular endothelial function is maintained. The second case was a 61 year-old male with a decreased FMD value of 


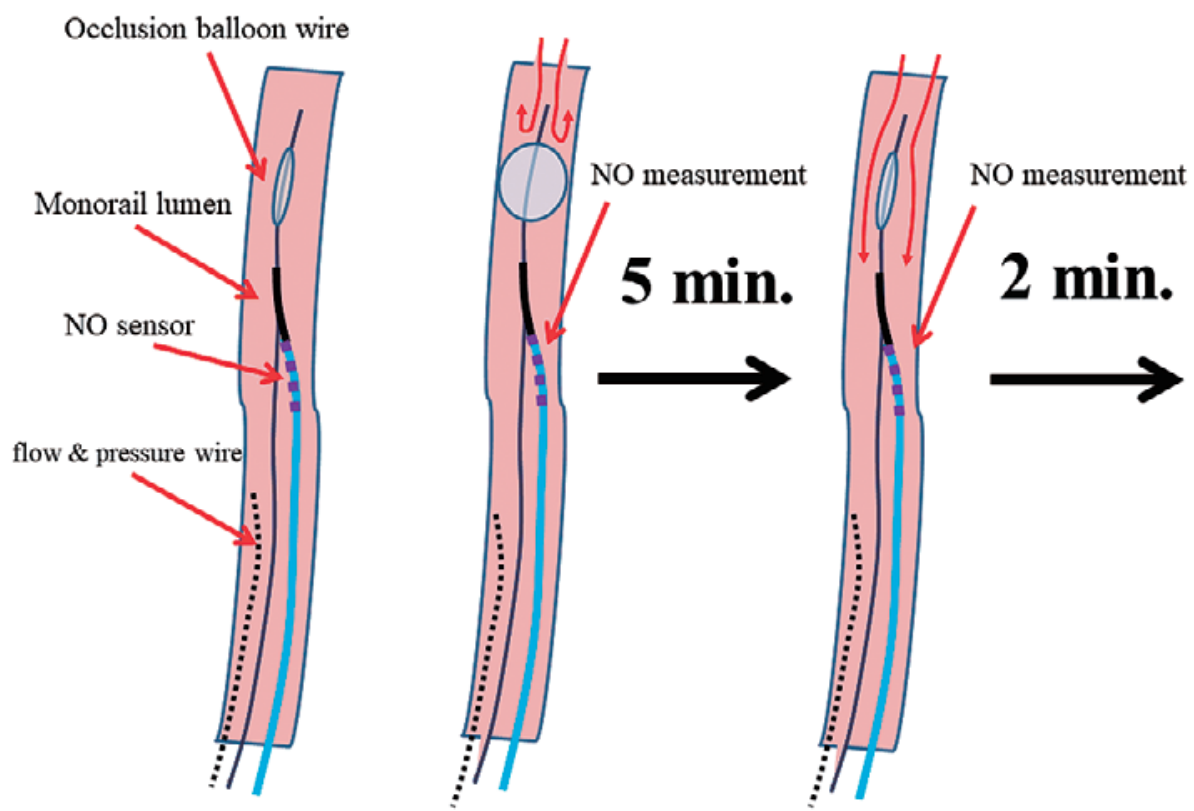

Figure 2. Direct-estimation of circulating NO kinetics using the NO sensor catheter system during ischemia/reactive hyperemia in the forearm artery.

$2.6 \%$, in whom the baseline circulating NO level was high, $80.6 \mathrm{nM}$. Despite the occlusion of the vessel by balloon inflation, the NO level increased only slightly to $81.8 \mathrm{nM}$ (a $1.4 \%$ increase). Moreover, there was no significant change in the NO level following deflation of the balloon (Figure 3-B). We can see that the baseline NO level was already elevated, although its mechanism is unclear, and speculate that there was no capacity for it to increase further. The NO kinetics, as described in the first case, might be absent in cases in which the vascular endothelial function is extremely impaired; although the precise mechanisms responsible for the differences between cases with and without vascular endothelial dysfunction remain to be elucidated.

\section{Clinical implications and limitation of current system}

In our experiments, we attempted to reproduce ischemia/ reactive hyperemia using an original model in which blood flow was blocked by balloon inflation and restored following balloon deflation, as an alternative to the inflation/deflation of forearm cuff in the FMD procedure. Although there is a procedural difference in the ischemia/reactive hyperemia models between our experiment and FMD procedure, i.e., vessel compression or luminal occlusion, we used this model to observe real-time dynamics of blood NO levels during the ischemia/reactive hyperemia maneuver. As a result, in our first case with normal vascular endothelial function based on the FMD, we observed an immediate transient increase in NO levels after occlusion of the blood flow and a gradual secondary increase in NO levels after restoration of the blood flow. We consider that the secondary response of the NO increase might correspond to the FMD response.
Therefore, we suggest that the balloon inflation/deflation model could reproduce ischemia/reactive hyperemia, similar to FMD. However, the current catheter-based system for the measurement of circulating NO appears to have many potential limitations for the real-time assessment of blood NO dynamics. First, even if the secondary response of the NO level represents endothelial function, this response appears to be attenuated, compared with the FMD response, as shown in our first case (5.7\% vs 6.9\%). As such, our approach to evaluate vascular endothelial function through the assessment of circulating NO kinetics with the NO sensor catheter would be less sensitive, compared with the FMD. A fundamental question arises as to how much of the endothelium-derived NO is released into blood stream; although it is certain that it diffuses into the vessel wall and relaxes the vascular smooth muscles. Furthermore, it is uncertain whether the change in blood NO represents a role in the endothelium-dependent regulation of vascular tone. Secondly, even if the NO is released into the blood stream, with respect to fluid dynamics of the blood vessel, it is unclear whether the NO concentration would be identical between the central stream and the marginal stream. In addition, it remains to be determined whether the NO sensor is able to detect NO in plasma vs. that in red blood cells, or both.

It is well known that red-blood cell hemoglobin transfers NO to peripheral tissue as a transporter. The endothelial NO, that is released to vascular cavity, momentarily combines with an iron $\left(\mathrm{Fe}^{2+}\right)$ of hemoglobin and is scavenged immediately ${ }^{14)}$, so it still remains unclear how much the circulating NO represents endothelium-released NO. We hypothesized that endothelium-released NO into the blood stream may be detected more precisely in the marginal blood stream close to the endothelium. However, with the current NO sensor 


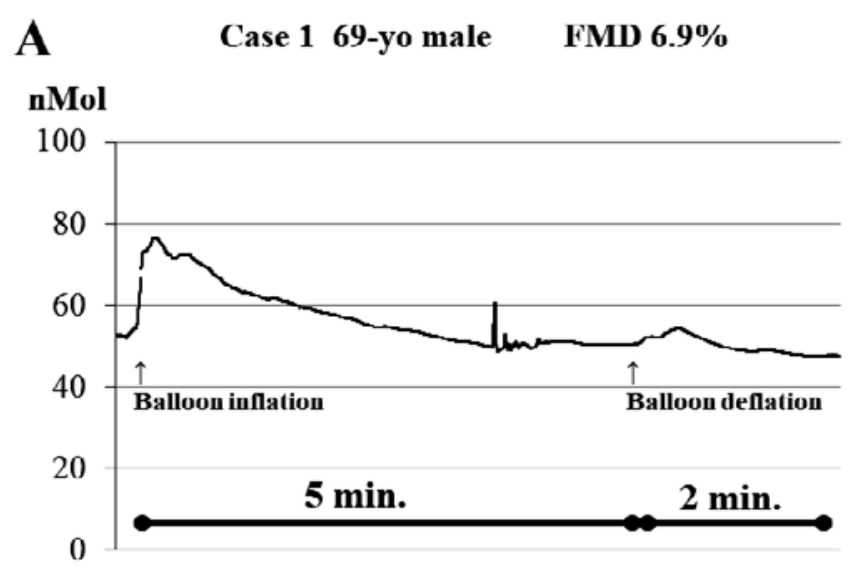

B

Case 2 61-yo male FMD 2.6\%

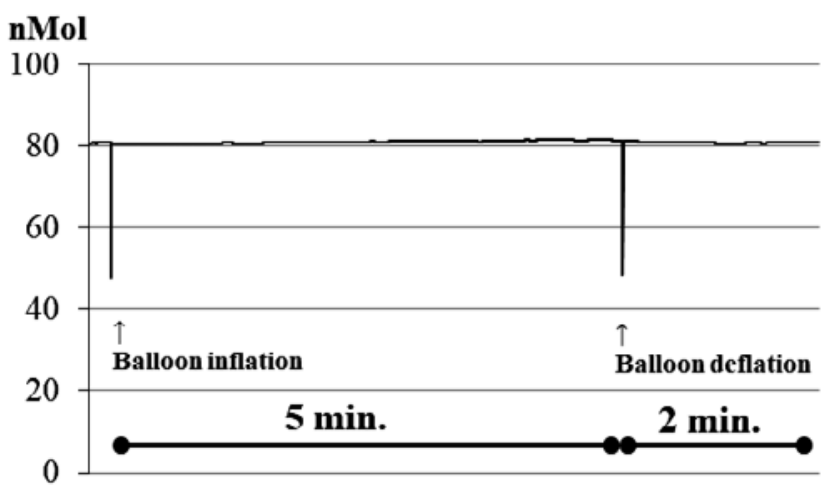

Figure 3. Circulating NO kinetics during ischemia/reactive hyperemia in the forearm artery. A. A 69 year-old male with a normal FMD value $(6.9 \%)$. The baseline NO level was $52.2 \mathrm{nM}$. After occlusion of the vessel by balloon inflation, the NO level immediately increased to the peak value of $76.4 \mathrm{nM}$ and gradually recovered to the baseline level thereafter. Following balloon deflation, the NO level increased gradually and slightly again to $55.2 \mathrm{nM}$ and then recovered to the baseline level. B. A 61 year-old male with a decreased FMD value (2.6\%). The baseline circulating NO level was $80.6 \mathrm{nM}$. Despite vessel occlusion by balloon inflation, the NO level increased only slightly to $81.8 \mathrm{nM}$. Moreover, there was no noteworthy change in the NO level following deflation of the balloon.

catheter system, it is not possible to determine where the catheter-tip NO sensor is positioned, in the central stream or in the marginal stream. Thus, we are now working to improve the system to detect NO levels in the marginal stream as close as possible to the endothelium. In addition to above mentioned limitations, there are many other problems to be resolved in the catheter-based NO sensor system. However, it would be possible to elucidate more precisely the mechanism of NO-dependent vasodilation and its response to various vasoactive drugs, if we can assess the circulating NO kinetics. We believe that when the system is successfully improved, the clinical application of the NO sensor catheter will provide us with novel information regarding vascular failure.

\section{Conclusion}

We describe a novel approach to evaluate the vascular endothelial response to ischemia/reactive hyperemia based upon blood NO kinetics using a catheter-based NO sensor with occlusion/release of blood flow with a balloon. Although this system currently has many problems that remain to be resolved, we believe that when it is successfully improved, the clinical application of this system will provide with novel information regarding vascular endothelial function.

Conflicts of Interest

There is no conflict of interest to disclose.

\section{References}

1. Inoue $T$, Node $K$. Vascular failure -a new clinical entity for vascular disease-. J Hypertens 2006; 24: 2121-30.

2. Corretti MC, Anderson TJ, Benjamin EJ, et al. Guidelines for the ultrasound assessment of endothelial-dependent flow-mediated vasodilatation of the brachial artery. J Am Coll Cardiol 2002; 39: 257-65.

3. Inoue T, Matsuoka H, Higashi Y, Ueda S, Sata M, Shimada K, et al. Flow-mediated vasodilation as a diagnostic modality for vascular failure. Hypertens Res 2008; 31: 2105-13.

4. Kamezaki F, Tsutsui M, Takahashi M, Sonoda S, Kubo T, Fujino Y, et al. Plasma levels of nitric oxide metabolites are markedly reduced in normotensive men with electrocardiographically determined left ventricular hypertrophy. Hypertension 2014; 64: 51622.

5. Wang F, Xiong R, Feng S, Lu X, Li H, Wang S. Association of circulating levels of ADMA with carotid intima-media thickness in patients with CKD: a systematic review and meta-analysis. Kidney Blood Press Res 2018; 43: 25-33.

6. Mochizuki S, Himi N, Miyasaka T, Nakamoto H, Takemoto M, Hirano $\mathrm{K}$, et al. Evaluation of basic performance and applicability of a newly developed in vivo nitric oxide sensor. Physiol Meas 2002; 23: 261-8.

7. Mochizuki S, Miyasaka T, Goto M, Ogasawara Y, Yada T, Akiyama $M$, et al. Measurement of acetylcholine-induced endothelium-derived nitric oxide in aorta using a newly developed catheter-type nitric oxide sensor. Biochem Biophy Res Commun 2003; 306: 505-8.

8. Neishi Y, Mochizuki S, Miyasaka T, Kawamoto T, Kume T, Sukmawan R, et al. Evaluation of bioavailability of nitric oxide in coronary circulation by direct measurement of plasma nitric oxide concentration. Proc Natl Acad Sci USA 2005; 102: 11456-61.

9. Imanishi T, Kuroi A, Ikejima H, Mochizuki S, Goto M, Akasaka T. Evaluation of pharmacological modulation of nitroglycerininduced impairment of nitric oxide bioavailability by a cathetertype nitric oxide sensor. Circ J 2007; 71: 1473-9.

10. Imanishi $T$, Ikejima H, Tsujioka H, Kuroi A, Kobayashi K, Muragaki $\mathrm{Y}$, et al. Addition of eplerenone to an angiotensin-converting enzyme inhibitor effectively improves nitric oxide bioavailability. Hypertension 2008; 51: 734-41.

11. Kume T, Kawamoto T, Okura H, Neishi Y, Hashimoto K, Hayashida A, et al. Evaluation of coronary endothelial function by catheter-type NO sensor in high-fat-diet-induced obese dogs. Circ J 2009; 73: 562-7.

12. Takarada S, Imanishi T, Goto M, Mochizuki S, Ikejima H, Tsujioka $\mathrm{H}$, et al. First evaluation of real-time nitric oxide changes in 
the coronary circulation in patients with non-ischaemic dilated cardiomyopathy using a catheter-type sensor. Eur Heart J 2010; 31: 2862-70.

13. Vogel RA. Measurement of endothelial function by brachial artery flow-mediated vasodilatation. Am J Cardiol 2001; 88: 31E-4E.

14. McMahon TJ, Moon RE, Luschinger BP, Carraway MS, Stone AE, Stolp BW, et al. Nitric oxide in the human respiratory cycle. Nature Medicine 2002; 8: 711-7. 